\title{
The Fourier spectral approximation for Kolmogorov-Spiegel-Sivashinsky equation
}

Xiaopeng Zhao, Bo Liu, and Peng Zhang 


\title{
THE FOURIER SPECTRAL APPROXIMATION FOR KOLMOGOROV-SPIEGEL-SIVASHINSKY EQUATION
}

\author{
XIAOPENG ZHAO, BO LIU, AND PENG ZHANG
}

Received 28 June, 2012

\begin{abstract}
In this paper, we consider the Fourier spectral approximation for numerically solving the Kolmogorov-Spiegel-Sivashinsky equation. The semi-discrete and fully discrete schemes are established. Moreover, the existence, uniqueness and the optimal error bound are also considered.

2010 Mathematics Subject Classification: 65M12; 65M70
\end{abstract}

Keywords: Kolmogorov-Spiegel-Sivashinsky equation, Fourier spectral approximation, existence, uniqueness, optimal error bound

\section{INTRODUCTION}

In this paper, we study the initial-boundary value problem for the Kolmogorov-Spiegel-Sivashinsky(KSS) equation

$$
\begin{aligned}
& u_{t}+k u_{x x x x}+\beta u+\gamma u_{x}^{2}+\alpha u_{x x}-\delta\left(u_{x}^{3}\right)_{x}=0,(x, t) \in Q_{T}, \\
& u_{x}(0, t)=u_{x}(1, t)=u_{x x x}(0, t)=u_{x x x}(1, t)=0, \\
& u(x, 0)=u_{0}(x), \quad x \in(0,1) .
\end{aligned}
$$

where $Q_{T}=(0,1) \times(0, T), k, \alpha, \beta, \gamma$ and $\delta$ are positive parameters.

Equation (1a), which was derived by Sivashinsky [10], is a fourth-order nonlinear parabolic equation, which models the effective negative viscosity in a certain direction $x$ of a large-scale flow. It is easy to check that if $\beta=0$ and $\delta=0$, the equation (1a) is the classical Kuramoto-Sivashinsky equation (see [1, 7,9, 11, 15]).

In [8], Nicolaenko presents mathematical and computational investigations of the finite dimensional behavior of the solutions for the above equation, and points out the existence of the global attractor and inertial manifold for the equation.

In [13], by discarding the linear damping term, Ünal and Suhubi obtained the KSS model's periodic, quasi-periodic and solitary wave solutions analytically to a certain degree of approximation. Melnikov analysis had also been carried out to identify

This paper is supported by the "Natural Science Foundation of Jiangsu Province of China for Young Scholar (No. BK20140130)" and the "Fundamental Research Funds for the Central Universities (No. JUSRP11407)". 
the homoclinic bifurcation. Transient spatiotemporal chaos had been observed. Ünal and Suhubi[12] also studied the group invariant solutions to the KSS equation. And a local analysis of the dynamical system obtained by the group theoretical means were performed by employing normal form analysis.

Recently, Guo and Wang[5] made a simple transform for the equation (1a). Differentiating the equation with respect to $x$ and setting $u_{x}=v$, they obtained

$$
v_{t}+k v_{x x x x}+\beta v+\gamma\left(v^{2}\right)_{x}+\alpha v_{x x}-\delta\left(v^{3}\right)_{x x}=f(x) .
$$

Therefore it is interesting to study the periodic BVP of (1.2) in multidimensional version. The authors first established the existence and uniqueness of the global solution, and then showed the existence of the global attractor, which has finite Hausdorff and fractal dimensions. Finally, they derived the Gevrey class regularity for the equation and constructed approximate inertial manifolds.

Fourier spectral approximations are essentially discretization methods for the approximate solution of partial differential equations. They have the natural advantage in keeping the physical properties of primitive problems. During the past years, many papers have already been published to study Fourier spectral method, for example $[3,6,16,17]$.

In this paper, we consider the Fourier spectral method for Kolmogorov-SpiegelSivashinsky equation (1a) with Neumann boundary condition (1b) and the initial condition (1c). Based on Sobolev's embedding theorem and some important inequalities, we obtain the error result $O\left((\Delta t)^{2}+N^{-s}\right)(s=2)$. Noticing that the existence of a solution locally in time is proved by the standard Picard iteration, global existence results are obtained by proving a priori estimates for the appropriate norms of $u(x, t)$. Adjusted to our needs, similar to the proof in $[4,18]$, the following results on global existence and uniqueness of solution to problem (1)-(3) are given in the following form:

Theorem 1. Assume that $u_{0} \in H_{E}^{2}(0,1)=\left\{w ; w \in H^{2}, w_{x}(0, t)=w_{x}(1, t)=0\right\}$. Then there exists a unique global solution $u(x, t)$ such that

$$
u(x, t) \in L^{\infty}\left(0, T ; H_{E}^{2}(0,1)\right) \bigcap L^{2}\left(0, T ; H^{4}(0,1)\right) .
$$

This paper is organized as follows. In the next section, we consider a semi-discrete Fourier spectral approximation, prove its existence and uniqueness of the numerical solution and derive the error bound. In Section 3, we consider the full-discrete approximation for problem (1). Furthermore, we prove convergence to the solution of the associated continuous problem. In Section 4, some numerical experiments which confirm our results are performed. In the last section, conclusions are given.

Throughout this paper, we denote the $L^{2}, L^{p}, L^{\infty}, H^{k}$ norms in $(0,1)$ simply by $\|\cdot\|,\|\cdot\|_{p},\|\cdot\|_{\infty}$ and $\|\cdot\|_{H^{k}}$. 


\section{SEMI-DISCRETE APPROXIMATION}

In this section, we consider the semi-discrete approximation for problem (1). First of all, we recall some basic results on the Fourier spectral method which will be used throughout this paper. For any integer $N>0$, we introduce the finite dimensional subspace of $H_{E}^{2}(0,1)$ :

$$
S_{N}=\operatorname{span}\{\cos k \pi x ; k=0,1, \cdots, N\} .
$$

Let $P_{N}: L^{2}(0,1) \rightarrow S_{N}$ be an orthogonal projecting operator which satisfies:

$$
\left(u-P_{N} u, v\right)=0, \forall v \in S_{N} .
$$

For the operator $P_{N}$, we have the following result (see $\left.[2,16]\right)$ :

(B1) $P_{N}$ commutes with derivation on $H_{E}^{2}(0,1)$, i.e.,

$$
P_{N} u_{x x}=\left(P_{N} u\right)_{x x}, \forall u \in H_{E}^{2}(0,1) .
$$

Using the same method as previous papers $[2,14]$, we can obtain the following result (B2) for problem (1):

(B2) For any real $0 \leq \mu \leq 2$, there is a constant $c$, such that

$$
\left\|u-P_{N} u\right\|_{\mu} \leq c N^{\mu-2}\left\|u_{x x}\right\|, \quad \forall u \in H_{E}^{2}(0,1) .
$$

We define the Fourier spectral approximation for problem (1): Find $u_{N}(t)=$ $\sum_{j=1}^{N} a_{j}(t) \cos j \pi x \in S_{N}$ such that

$$
\begin{aligned}
& \left(\frac{\partial u_{N}}{\partial t}, v_{N}\right)+k\left(u_{N x x}, v_{N x x}\right)+\beta\left(u_{N}, v_{N}\right)+\gamma\left(u_{N x}^{2}, v_{N}\right)-\alpha\left(u_{N x}, v_{N x}\right) \\
& -\delta\left(\left(u_{N x}^{3}\right)_{x}, v_{N}\right)=0, \quad \forall v_{N} \in S_{N},
\end{aligned}
$$

for all $T \geq 0$ with $u_{N}(0)=P_{N} u_{0}$.

Now, we are going to establish the existence and uniqueness of the Fourier spectral approximation solution $u_{N}(t)$ for all $T \geq 0$.

Lemma 1. Let $u_{0} \in H_{E}^{2}(0,1)$, then (2.2) has a unique solution $u_{N}(t)$ satisfying the following inequalities:

$$
\left\|u_{N}(t)\right\|_{H_{E}^{2}}^{2} \leq c_{1}\left\|u_{0}\right\|_{H_{E}^{2}}^{2}, \int_{0}^{T}\left\|u_{N}(\tau)\right\|_{H_{E}^{4}}^{2} d \tau \leq c_{1}^{\prime}\left\|u_{0}\right\|_{H_{E}^{2}}^{2},
$$

where $c_{1}$ and $c_{1}^{\prime}$ are positive constants depend only on $\alpha, \beta, \gamma, \delta, T$ and $\left\|u_{0}\right\|_{H_{E}^{2}}$, independents of $N$.

Proof. Set $v_{N}=\cos j \pi x$ in (2.2) for each $j(1 \leq j \leq N)$ to obtain

$$
\frac{d}{d t} a_{j}(t)=f_{j}\left(a_{1}(t), a_{2}(t), \cdots, a_{N}(t)\right), \quad j=1,2, \cdots, N,
$$


where all $f_{j}: \mathbb{R}^{N} \rightarrow \mathbb{R}(1 \leq j \leq N)$ are smooth and locally Lipschitz continuous. Note that $u_{N}(0)=P_{N} u_{0}$. Therefore

$$
a_{j}(0)=\left(u_{0}, \cos j \pi x\right), \quad j=1,2, \cdots, N .
$$

Using the theory of initial-value problems of the ordinary differential equations, there is a time $T_{N}>0$ such that the initial-value problem (2.4)-(2.5) has a unique smooth solution $\left(a_{1}(t), a_{2}(t), \cdots, a_{N}(t)\right)$ for $t \in\left[0, T_{N}\right]$. Therefore, there are three steps for us to prove the lemma:

Step 1. Setting $v_{N}=u_{N}$ in (2.2), we obtain

$$
\frac{1}{2} \frac{d}{d t}\left\|u_{N}\right\|^{2}+k\left\|u_{N x x}\right\|^{2}+\beta\left\|u_{N}\right\|^{2}+\delta\left\|u_{N x}\right\|_{4}^{4}=-\gamma\left(u_{N x}^{2}, u_{N}\right)-\alpha\left(u_{N x x}, u_{N}\right) .
$$

Noticing that

and

$$
-\gamma\left(u_{N x}^{2}, u_{N}\right) \leq \delta\left\|u_{N x}\right\|_{4}^{4}+\frac{\gamma^{2}}{4 \delta}\left\|u_{N}\right\|^{2}
$$

$$
-\alpha\left(u_{N x x}, u_{N}\right) \leq \frac{k}{2}\left\|u_{N x x}\right\|^{2}+\frac{\alpha^{2}}{2 k}\left\|u_{N}\right\|^{2} .
$$

Hence, by a simple calculation, we get

$$
\frac{d}{d t}\left\|u_{N}\right\|^{2}+k\left\|u_{N x x}\right\|^{2}=\left(\frac{\gamma^{2}}{2 \delta}+\frac{\alpha^{2}}{k}-2 \beta\right)\left\|u_{N}\right\|^{2} .
$$

Using Gronwall's inequality, we deduce that

$$
\left\|u_{N}\right\|^{2} \leq e^{\left(\frac{\gamma^{2}}{2 \delta}+\frac{\alpha^{2}}{k}-2 \beta\right) t}\left\|u_{N}(0)\right\|^{2} \leq e^{\left(\frac{\gamma^{2}}{2 \delta}+\frac{\alpha^{2}}{k}-2 \beta\right) T}\left\|u_{0}\right\|^{2}, \quad \forall t \in[0, T] .
$$

Integrating (2.6) from 0 to $T$, we obtain

$$
\begin{aligned}
\int_{0}^{T}\left\|u_{N x x}\right\|^{2} d t & \leq \frac{1}{k}\left[\left(\frac{\gamma^{2}}{2 \delta}+\frac{\alpha^{2}}{k}-2 \beta\right) \int_{0}^{T}\left\|u_{N}\right\|^{2} d t+\left\|u_{N}(0)\right\|\right] \\
& \leq \frac{1}{k}\left[\left(\frac{\gamma^{2}}{2 \delta}+\frac{\alpha^{2}}{k}-2 \beta\right) e^{\left(\frac{\gamma^{2}}{2 \delta}+\frac{\alpha^{2}}{k}-2 \beta\right) T}+1\right]\left\|u_{0}\right\|^{2} .
\end{aligned}
$$

Step 2. Setting $v_{N}=-u_{N x x}$ in (2.2), we obtain

$$
\begin{aligned}
& \frac{1}{2} \frac{d}{d t}\left\|u_{N x}\right\|^{2}+k\left\|u_{N x x x}\right\|^{2}+\beta\left\|u_{N x}\right\|^{2}-\gamma\left(u_{N x}^{2}, u_{N x x}\right) \\
& -\alpha\left\|u_{N x x}\right\|^{2}+3 \delta\left(u_{N x}^{2} u_{N x x}, u_{N x x}\right)=0 .
\end{aligned}
$$

Noticing that

$$
\gamma\left(u_{N x}^{2}, u_{N x x}\right)=0, \quad \delta\left(u_{N x}^{2} u_{N x x}, u_{N x x}\right) \geq 0, \quad \alpha\left\|u_{N x x}\right\|^{2}=-\alpha\left(u_{N x}, u_{N x x x}\right) .
$$

Then, we have

$$
\frac{1}{2} \frac{d}{d t}\left\|u_{N x}\right\|^{2}+k\left\|u_{N x x x}\right\|^{2}+\beta\left\|u_{N x}\right\|^{2} \leq \alpha\left\|u_{N x x}\right\|^{2} \leq \frac{k}{2}\left\|u_{N x x x}\right\|^{2}+\frac{\alpha^{2}}{2 k}\left\|u_{N x}\right\|^{2},
$$


that is

$$
\frac{d}{d t}\left\|u_{N x}\right\|^{2}+k\left\|u_{N x x x}\right\|^{2} \leq\left(\frac{\alpha^{2}}{k}-2 \beta\right)\left\|u_{N x}\right\|^{2} .
$$

Using Gronwall's inequality, we get

$$
\left\|u_{N x}\right\|^{2} \leq e^{\left(\frac{\alpha^{2}}{k}-2 \beta\right) t}\left\|u_{N x}(0)\right\|^{2} \leq e^{\left(\frac{\alpha^{2}}{k}-2 \beta\right) T}\left\|u_{x 0}\right\|^{2}, \quad \forall t \in[0, T] .
$$

Integrating (2.10) from 0 to $T$, we obtain

$$
\begin{aligned}
\int_{0}^{T}\left\|u_{N x x x}\right\|^{2} d t & \leq \frac{1}{k}\left[\left(\frac{\alpha^{2}}{k}-2 \beta\right) e^{\left(\frac{\alpha^{2}}{k}-2 \beta\right) T}\left\|u_{x 0}\right\|^{2}+\left\|u_{N x}(0)\right\|^{2}\right] \\
& \leq \frac{1}{k}\left[\left(\frac{\alpha^{2}}{k}-2 \beta\right) e^{\left(\frac{\alpha^{2}}{k}-2 \beta\right) T}+1\right]\left\|u_{x 0}\right\|^{2} .
\end{aligned}
$$

Step 3. Setting $v_{N}=u_{N x x x x}$ in (2.2), we obtain

$$
\begin{aligned}
& \frac{1}{2} \frac{d}{d t}\left\|u_{N x x}\right\|^{2}+k\left\|u_{N x x x x}\right\|^{2}+\beta\left\|u_{N x x}\right\|^{2} \\
& +\gamma\left(\left(u_{N x}\right)^{2}, u_{N x x x x}\right)+\alpha\left(u_{N x x}, u_{N x x x x}\right)-3 \delta\left(\left(u_{N x}\right)^{2} u_{N x x}, u_{N x x x x}\right)=0 .
\end{aligned}
$$

By Nirenberg's inequality, we deduce that

and

$$
\begin{aligned}
\left\|u_{N x}\right\|_{8}^{2} & \leq c^{\prime}\left\|u_{N x x x x}\right\|^{\frac{1}{4}}\left\|u_{N x}\right\|^{\frac{7}{4}}, \\
\left\|u_{N x}\right\|_{4}^{4} & \leq c^{\prime}\left\|u_{N x x x x}\right\|^{\frac{1}{3}}\left\|u_{N x}\right\|^{\frac{11}{3}},
\end{aligned}
$$

$$
\left\|u_{N x x}\right\|_{4} \leq c^{\prime}\left\|u_{N x x x x}\right\|^{\frac{5}{12}}\left\|u_{N x}\right\|^{\frac{7}{12}}
$$

where $c^{\prime}$ is a positive constant dependents only on the domain. Hence,

$$
\begin{aligned}
3 \delta\left(\left(u_{N x}\right)^{2} u_{N x x}, u_{N x x x x}\right) & \leq 3 \delta\left\|u_{N x}\right\|_{8}^{2}\left\|u_{N x x}\right\|\left\|u_{N x x x x}\right\| \\
& \leq 3 \delta\left(c^{\prime}\right)^{2}\left\|u_{N x x x x}\right\|^{\frac{5}{3}}\left\|u_{N x}\right\|^{\frac{7}{3}} \leq \frac{k}{6}\left\|u_{N x x x x}\right\|^{2}+c_{2},
\end{aligned}
$$

where $c_{2}$ is a positive constant depends only on $k, \alpha, \beta, \delta, T$ and $\left\|u_{0}\right\|_{H^{1}}$, independents of $N$. On the other hand, we have

$$
-\gamma\left(\left(u_{N x}\right)^{2}, u_{N x x x x}\right) \leq \frac{k}{12}\left\|u_{N x x x x}\right\|^{2}+\frac{3 \gamma^{2}}{k}\left\|u_{N x}\right\|_{4}^{4} \leq \frac{k}{6}\left\|u_{N x x x x}\right\|^{2}+c_{3},
$$

where $c_{3}$ is a positive constant depends only on $k, \alpha, \beta, T$ and $\left\|u_{0}\right\|_{H^{1}}$, independents of $N$. We also have

$$
-\alpha\left(u_{N x x}, u_{N x x x x}\right) \leq \frac{k}{6}\left\|u_{N x x x x}\right\|^{2}+\frac{3 \alpha^{2}}{2 k}\left\|u_{N x x}\right\|^{2} .
$$

Summing up, we get

$$
\frac{d}{d t}\left\|u_{N x x}\right\|^{2}+k\left\|u_{N x x x x}\right\|^{2} \leq\left(\frac{3 \alpha^{2}}{k}-2 \beta\right)\left\|u_{N x x}\right\|^{2}+2\left(c_{2}+c_{3}\right) .
$$


Using Gronwall's inequality, we obtain

$$
\begin{aligned}
\left\|u_{N x x}\right\|^{2} & \leq e^{\left(\frac{3 \alpha^{2}}{k}-2 \beta\right) t}\left(\left\|u_{N x x}(0)\right\|^{2}+2\left(c_{2}+c_{3}\right) t\right) \\
& \leq e^{\left(\frac{3 \alpha^{2}}{k}-2 \beta\right) T}\left(\left\|u_{x x 0}\right\|^{2}+2\left(c_{2}+c_{3}\right) T\right)=c_{4}, \quad \forall t \in[0, T],
\end{aligned}
$$

where $c_{4}$ is a positive constant depends only on $k, \alpha, \beta, \gamma, \delta, T$ and $\left\|u_{0}\right\|_{H^{2}}$, independents of $N$. Integrating (2.13) from 0 to $T$, we obtain

$$
\begin{aligned}
& \int_{0}^{T}\left\|u_{N x x x x}\right\|^{2} d t \\
\leq & \frac{1}{k}\left[\left(\frac{3 \alpha^{2}}{k}-2 \beta\right) \int_{0}^{T}\left\|u_{N x x}\right\|^{2} d t+2\left(c_{2}+c_{3}\right) T+\left\|u_{N x x}(0)\right\|^{2}\right] \\
\leq & \frac{1}{k}\left[\left(\frac{3 \alpha^{2}}{k}-2 \beta\right) c_{4}^{2} T+2\left(c_{2}+c_{3}\right) T+\left\|u_{x x 0}\right\|^{2}\right]=c_{5} .
\end{aligned}
$$

where $c_{5}$ is a positive constant depends only on $k, \alpha, \beta, \gamma, \delta, T$ and $\left\|u_{0}\right\|_{H^{2}}$, independents of $N$.

Combining (2.7), (2.8), (2.11), (2.12), (2.14), (2.15) together, we get the result of Lemma 1.

Hence, we have the following theorem on the existence and uniqueness of global solution for problem (2.2).

Theorem 2. Let $u_{0} \in H_{E}^{2}(0,1)$, then for any $T>0$, problem (2.2) admits a unique global solution $u_{N}(x, t)$, such that

$$
u_{N}(x, t) \in L^{\infty}\left(0, T ; H_{E}^{2}(0,1)\right) \bigcap L^{2}\left(0, T ; H^{4}(0,1)\right) .
$$

Proof. We are going to apply the Leray-Schauder fixed point theorem to complete the proof. Define the linear space

$$
\begin{aligned}
X= & \left\{u_{N} \in L^{\infty}\left(0, T ; H_{E}^{2}(0,1)\right) \bigcap L^{2}\left(0, T ; H^{4}(0,1)\right) ;\right. \\
& \left.u_{N x}(0, t)=u_{N x}(1, t)=0, u_{N}(x, 0)=u_{0}(x)\right\} .
\end{aligned}
$$

Clearly, $X$ is a Banach space. Define the associated operator $T$,

$$
T: X \rightarrow X, \quad u_{N} \mapsto w,
$$

where $w$ is determined by the following linear problem:

$$
\begin{aligned}
& \frac{\partial w}{\partial t}+k w_{x x x x}+\beta w+\alpha w_{x x}=-\gamma u_{N x}^{2}+\delta\left(u_{N x}^{3}\right)_{x}, \quad x \in(0,1), \\
& w_{x}(0, t)=w_{x}(1, t)=w_{x x x}(0, t)=w_{x x x}(1, t)=0, t \in(0, T), \\
& w(x, 0)=u_{0}(x) .
\end{aligned}
$$


Form the discussions in Lemmas 1 and by the contraction mapping principle, $T$ has a unique fixed point $u_{N}$, which is the desired solution of problem (1). Since the proof of the uniqueness of the solution is easy, we omit it here. Then, Theorem 2 is proved.

Now, we give the following theorem.

Theorem 3. Let $u_{0} \in H_{E}^{2}(0,1), u(x, t)$ is the solution of problem (1) and $u_{N}(x, t)$ is the solution of semi-discrete approximation (2.2). Then, there exists a constant $c$, depends on $k, \alpha, \beta, \gamma, \delta, T$ and $\left\|u_{0}\right\|_{H_{E}^{2}}$, independent of $N$, such that

$$
\left\|u(x, t)-u_{N}(x, t)\right\| \leq c\left(N^{-2}+\left\|u_{0}-u_{N}(0)\right\|\right) .
$$

Proof. Denote $\eta_{N}=u(t)-P_{N} u(t)$ and $e_{N}=P_{N} u(t)-u_{N}(t)$. It then follows from (1a) and (2.2) that

$$
\begin{aligned}
& \left(e_{N t}, v_{N}\right)+k\left(e_{N x x}, v_{N x x}\right)+\beta\left(e_{N}, v_{N}\right)+\gamma\left(u_{x}^{2}-u_{N x}^{2}, v_{N}\right) \\
& +\alpha\left(e_{N}, v_{N x x}\right)+\delta\left(u_{x}^{3}-u_{N x}^{3}, v_{N x}\right)=0, \forall v_{N} \in S_{N} .
\end{aligned}
$$

Setting $v_{N}=e_{N}$ in (2.16), we derive that

$$
\begin{aligned}
& \frac{1}{2} \frac{d}{d t}\left\|e_{N}\right\|^{2}+k\left\|e_{N x x}\right\|^{2}+\beta\left\|e_{N}\right\|^{2} \\
= & -\gamma\left(u_{x}^{2}-u_{N x}^{2}, e_{N}\right)-\alpha\left(e_{N}, e_{N x x}\right)-\delta\left(u_{x}^{3}-u_{N x}^{3}, e_{N x}\right) .
\end{aligned}
$$

By Theorem 1, we have

$$
\|u(x, t)\|_{H_{E}^{2}} \leq c\left(k, \alpha, \beta, \gamma, \delta,\left\|u_{0}\right\|_{H_{E}^{2}}\right),
$$

Using Sobolev's embedding theorem, we get

$$
\|u(x, t)\|_{W^{1, \infty}} \leq c\left(k, \alpha, \beta, \gamma, \delta,\left\|u_{0}\right\|_{H^{2}}\right) .
$$

We also have

$$
\left\|e_{N}\right\|_{\infty}^{2} \leq c\left\|e_{N}\right\|_{H^{1}}^{2} \leq c\left(\left\|e_{N}\right\|^{2}+\left\|e_{N x}\right\|^{2}\right) \leq c^{\prime}\left(\left\|e_{N}\right\|^{2}+\left\|e_{N x x}\right\|^{2}\right),
$$

and

$$
\left\|e_{N x}\right\|_{\infty}^{2} \leq c\left\|e_{N}\right\|_{H_{E}^{2}}^{2} \leq c\left(\left\|e_{N}\right\|^{2}+\left\|e_{N x}\right\|^{2}+\left\|e_{N x x}\right\|^{2}\right) \leq c^{\prime}\left(\left\|e_{N}\right\|^{2}+\left\|e_{N x x}\right\|^{2}\right) .
$$

Then

$$
\begin{aligned}
& -\gamma\left(u_{x}^{2}-u_{N x}^{2}, e_{N}\right) \\
= & -\gamma\left(\left(u_{x}+u_{N x}\right)\left(e_{N x}+\eta_{N x}\right), e_{N}\right) \\
= & \gamma\left(e_{N}+\eta_{N},\left(u_{x}+u_{N x}\right) e_{N x}+\left(u_{x x}+u_{N x x}\right) e_{N}\right) \\
\leq & \gamma\left(\left\|e_{N}\right\|\left\|e_{N x}\right\|\left\|u_{x}+u_{N x}\right\|_{\infty}+\left\|\eta_{N}\right\|\left\|e_{N x}\right\|\left\|u_{x}+u_{N x}\right\|_{\infty}\right. \\
& \left.+\left\|e_{N}\right\|\left\|u_{x x}+u_{N x x}\right\|\left\|e_{N}\right\|_{\infty}+\left\|\eta_{N}\right\|\left\|u_{x x}+u_{N x x}\right\|\left\|e_{N}\right\|_{\infty}\right) \\
\leq & \frac{k}{6}\left\|e_{N x x}\right\|^{2}+c_{6}\left(\left\|e_{N}\right\|^{2}+\left\|\eta_{N}\right\|^{2}\right),
\end{aligned}
$$


and

$$
\begin{aligned}
& -\delta\left(u_{x}^{3}-u_{N x}^{3}, e_{N x}\right) \\
= & -\delta\left(\left(e_{N x}+\eta_{N x}\right)\left(u_{x}^{2}+u_{x} u_{N x}+u_{N x}^{2}\right), e_{N x}\right) \\
= & \delta\left(e_{N}+\eta_{N},\left(u_{x}^{2}+u_{x} u_{N x}+u_{N x}^{2}\right) e_{N x x}\right) \\
& \delta\left(e_{N}+\eta_{N},\left(2 u_{x} u_{x x}+u_{x} u_{N x x}+u_{x x} u_{N x}+2 u_{N x x}\right) e_{N x}\right) \\
\leq & \delta\left(\left\|e_{N}\right\|+\left\|\eta_{N}\right\|\right)\left\|e_{N x x}\right\|\left\|u_{x}^{2}+u_{x} u_{N x}+u_{N x}^{2}\right\|_{\infty} \\
& +\delta\left(\left\|e_{N}\right\|+\left\|\eta_{N}\right\|\right)\left\|2 u_{x} u_{x x}+u_{x} u_{N x x}+u_{x x} u_{N x}+2 u_{N x x}\right\|\left\|e_{N x}\right\|_{\infty} \\
\leq & \frac{k}{6}\left\|e_{N x x}\right\|^{2}+c_{7}\left(\left\|e_{N}\right\|^{2}+\left\|\eta_{N}\right\|^{2}\right),
\end{aligned}
$$

where $c_{6}$ and $c_{7}$ are positive constants depends only on $k, \alpha, \beta, \gamma, \delta, T$ and $\left\|u_{0}\right\|_{H^{2}}$, independents of $N$. We also have

$$
\alpha\left(e_{N}, e_{N x x}\right) \leq \frac{k}{6}\left\|e_{N x x}\right\|^{2}+\frac{3 \alpha^{2}}{2 k}\left\|e_{N}\right\|^{2} .
$$

Summing up, we deduce that

$$
\frac{d}{d t}\left\|e_{N}\right\|^{2}+k\left\|e_{N x x}\right\|^{2} \leq\left(2 c_{6}+2 c_{7}+\frac{3 \alpha^{2}}{k}-2 \beta\right)\left\|e_{N}\right\|^{2}+\left(2 c_{6}+2 c_{7}\right)\left\|\eta_{N}\right\|^{2} .
$$

Noticing that

$$
\left\|\eta_{N}\right\|^{2} \leq c N^{-4}\left\|u_{x x}\right\|^{2} \leq c_{8}\left(k, \alpha, \beta, \gamma, \delta, T,\left\|u_{0}\right\|_{H^{2}}\right) N^{-4} .
$$

Therefore

$$
\frac{d}{d t}\left\|e_{N}\right\|^{2}+k\left\|e_{N x x}\right\|^{2} \leq\left(2 c_{6}+2 c_{7}+\frac{3 \alpha^{2}}{k}-2 \beta\right)\left\|e_{N}\right\|^{2}+c_{8}\left(2 c_{6}+2 c_{7}\right) N^{-4} .
$$

Using Gronwall's inequality, we complete the proof.

\section{FULLY DISCRETE SCHEME}

Let $\Delta t$ be the time-step, the full-discretization spectral method for problem (1) is read as: find $u_{N}^{j} \in S_{N}(j=0,1,2, \cdots, N)$ such that

$$
\begin{aligned}
& \left(\frac{u_{N}^{j+1}-u_{N}^{j}}{\Delta t}, v_{N}\right)+k\left(\bar{u}_{N x x}^{j+\frac{1}{2}}, v_{N x x}\right)+\beta\left(\bar{u}_{N}^{j+\frac{1}{2}}, v_{N}\right)+\gamma\left(\left(\bar{u}_{N x}^{j+\frac{1}{2}}\right)^{2}, v_{N}\right) \\
& +\alpha\left(\bar{u}_{N}^{j+\frac{1}{2}}, v_{N x x}\right)+\delta\left(\left(\bar{u}_{N x}^{j+\frac{1}{2}}\right)^{3}, v_{N x}\right)=0, \quad \forall v_{N} \in S_{N},
\end{aligned}
$$

with $u_{N}(0)=P_{N} u_{0}$, where $\bar{u}_{N}^{j+\frac{1}{2}}=\frac{1}{2}\left(u_{N}^{j}+u_{N}^{j+1}\right)$.

The solution $u_{N}^{j}$ has the following property: 
Lemma 2. Assume that $u_{0} \in H_{E}^{2}(0,1)$. Suppose that $u_{N}^{j}$ is a solution of problem (3.1), then there exists positive constants $c_{9}, c_{10}$ depend only on $k, \alpha, \beta, \gamma, \delta, T$ and $\left\|u_{0}\right\|_{H_{E}^{2}}$, independent of $N$, such that

$$
\left\|u_{N}^{j}\right\|_{H_{E}^{2}} \leq c_{9},\left\|u_{N}^{j}\right\|_{W_{E}^{1, \infty}} \leq c_{10} .
$$

Proof. We can use the same method as Lemma 1 to prove this lemma. Since the proof is so easy, we omit it here.

In the following, we analyze the error estimates between the numerical solution $u_{N}^{j}$ and the exact solution $u\left(t_{j}\right)$. According to the properties of the projection operator $P_{N}$, we only need to analyze the error between $P_{N} u\left(t_{j}\right)$ and $u_{N}^{j}$. Denoted by $u^{j}=u\left(t_{j}\right), e^{j}=P_{N} u^{j}-u_{N}^{j}$ and $\eta^{j}=u^{j}-P_{N} u^{j}$. Therefore

$$
u^{j}-u_{N}^{j}=\eta^{j}+e^{j} .
$$

If no confusion occurs, we denote the average of the two instant errors $e^{n}$ and $e^{n+1}$ by $\bar{e}^{n+\frac{1}{2}}$, where $\bar{e}^{n+\frac{1}{2}}=\frac{e^{n}+e^{n+1}}{2}$. On the other hand, we let $\bar{\eta}^{j+\frac{1}{2}}=\frac{\eta^{j}+\eta^{j+1}}{2}$.

Firstly, we give the following error estimates for the full discretization scheme.

Lemma 3 (see [3]). For the instant errors $e^{j+1}$ and $e^{j}$, we have

$$
\begin{aligned}
\left\|e^{j+1}\right\|^{2} \leq & \left\|e^{j}\right\|^{2}+2 \Delta t\left(u_{t}\left(t_{j+\frac{1}{2}}\right)-\frac{u_{N}^{j+1}-u_{N}^{j}}{\Delta t}, \bar{e}^{j+\frac{1}{2}}\right) \\
& +\frac{1}{320}(\Delta t)^{4} \int_{t_{j}}^{t_{j+1}}\left\|u_{t t t}\right\|^{2} d t+\Delta t\left\|\bar{e}^{j+\frac{1}{2}}\right\|^{2} .
\end{aligned}
$$

of (1a) with $\bar{e}^{j+\frac{1}{2}}$, letting $t=t_{j+\frac{1}{2}}$, we obtain

$$
\begin{aligned}
& \left(u_{t}^{j+\frac{1}{2}}, \bar{e}^{j+\frac{1}{2}}\right)+\left(k u_{x x}^{j+\frac{1}{2}}, \bar{e}_{x x}^{j+\frac{1}{2}}\right)+\beta\left(u^{j+\frac{1}{2}}, \bar{e}^{j+\frac{1}{2}}\right) \\
& +\gamma\left(\left(\bar{u}_{x}^{j+\frac{1}{2}}\right)^{2}, \bar{e}^{j+\frac{1}{2}}\right)+\alpha\left(\bar{u}^{j+\frac{1}{2}}, \bar{e}_{x x}^{j+\frac{1}{2}}\right)+\delta\left(\left(u_{x}^{j+\frac{1}{2}}\right)^{3}, \bar{e}_{x}^{j+\frac{1}{2}}\right)=0
\end{aligned}
$$

Taking $v_{N}=\bar{e}^{n+\frac{1}{2}}$ in (3.1), we obtain

$$
\begin{aligned}
& \left(\frac{u_{N}^{j+1}-u_{N}^{j}}{\Delta t}, \bar{e}^{j+\frac{1}{2}}\right)+k\left(\bar{u}_{N x x}^{j+\frac{1}{2}}, \bar{e}_{x x}^{j+\frac{1}{2}}\right)+\beta\left(\bar{u}_{N}^{j+\frac{1}{2}}, \bar{e}^{j+\frac{1}{2}}\right) \\
& +\gamma\left(\left(\bar{u}_{N x}^{j+\frac{1}{2}}\right)^{2}, \bar{e}^{j+\frac{1}{2}}\right)+\alpha\left(\bar{u}_{N}^{j+\frac{1}{2}}, \bar{e}_{x x}^{j+\frac{1}{2}}\right)+\delta\left(\left(u_{N x}^{j+\frac{1}{2}}\right)^{3}, \bar{e}_{x}^{j+\frac{1}{2}}\right)=0
\end{aligned}
$$


Comparing the above two equations, we get

$$
\begin{aligned}
& \left(u_{t}^{j+\frac{1}{2}}-\frac{u_{N}^{j+1}-u_{N}^{j}}{\Delta t}, \bar{e}^{j+\frac{1}{2}}\right) \\
= & -k\left(u_{x x}^{j+\frac{1}{2}}-\bar{u}_{N x x}^{j+\frac{1}{2}}, \bar{e}_{x x}^{j+\frac{1}{2}}\right)-\beta\left(u^{j+\frac{1}{2}}-\bar{u}_{N}^{j+\frac{1}{2}}, \bar{e}^{j+\frac{1}{2}}\right)-\gamma\left(\left(u_{x}^{j+\frac{1}{2}}\right)^{2}\right. \\
& \left.-\left(\bar{u}_{N x}^{j+\frac{1}{2}}\right)^{2}, \bar{e}^{j+\frac{1}{2}}\right)-\alpha\left(u^{j+\frac{1}{2}}-\bar{u}_{N}^{j+\frac{1}{2}}, \bar{e}_{x x}^{j+\frac{1}{2}}\right)-\delta\left(\left(u_{x}^{j+\frac{1}{2}}\right)^{3}-\left(\bar{u}_{N x}^{j+\frac{1}{2}}\right)^{3}, \bar{e}_{x}^{j+\frac{1}{2}}\right) .
\end{aligned}
$$

Now, we investigate the error estimates of the five items in the right-hand side of previous equation.

Lemma 4. Assume that $u_{0} \in H_{E}^{2}(0,1), u$ is the solution for problem (1) and $u_{N}^{j}$ is the solution for problem (3.1). Then

$$
-k\left(u_{x x}^{j+\frac{1}{2}}-\bar{u}_{N x x}^{j+\frac{1}{2}}, \bar{e}_{x x}^{j+\frac{1}{2}}\right) \leq-\frac{k}{2}\left\|\bar{e}_{x x}^{j+\frac{1}{2}}\right\|^{2}+\frac{k(\Delta t)^{3}}{192} \int_{t_{j}}^{t_{j+1}}\left\|u_{x x t t}\right\|^{2} d t .
$$

Proof. Using Taylor's expansion, we obtain

$$
\begin{aligned}
u^{j} & =u^{j+\frac{1}{2}}-\frac{\Delta t}{2} u_{t}^{j+\frac{1}{2}}+\int_{t_{j}}^{t_{j+\frac{1}{2}}}\left(t-t_{j}\right) u_{t} d t, \\
u^{j+1} & =u^{j+\frac{1}{2}}+\frac{\Delta t}{2} u_{t}^{j+\frac{1}{2}}+\int_{t_{j+\frac{1}{2}}}^{t_{j+1}}\left(t_{j}-t\right) u_{t t} d t .
\end{aligned}
$$

Hence

$$
\frac{1}{2}\left(u^{j}+u^{j+1}\right)-u^{j+\frac{1}{2}}=\frac{1}{2}\left(\int_{t_{j}}^{t_{j+\frac{1}{2}}}\left(t-t_{j}\right) u_{t t} d t+\int_{t_{j+\frac{1}{2}}}^{t_{j+1}}\left(t_{j}-t\right) u_{t t} d t\right) .
$$

By Hölder's inequality, we have

$$
\begin{aligned}
& \left.\| u_{x x}^{j+\frac{1}{2}}-\frac{1}{2}\left(u_{x x}^{j}+u_{x x}^{j+1}\right)\right) \|^{2} \\
= & \frac{1}{4}\left(\left\|\left(\int_{t_{j}}^{t_{j+\frac{1}{2}}}\left(t-t_{j}\right) u_{t t} d t+\int_{t_{j+\frac{1}{2}}}^{t_{j+1}}\left(t_{j}-t\right) u_{t t} d t\right)_{x x}\right\|^{2}\right) \\
\leq & \frac{(\Delta t)^{3}}{96} \int_{t_{j}}^{t_{j+1}}\left\|u_{x x t t}\right\|^{2} d t .
\end{aligned}
$$


Noticing that $\left(\bar{\eta}_{x x}^{j+\frac{1}{2}}, \bar{e}_{x x}^{j+\frac{1}{2}}\right)=0$. Therefore

$$
\begin{aligned}
& -\left(u_{x x}^{j+\frac{1}{2}}-\bar{u}_{N x x}^{j+\frac{1}{2}}, \bar{e}_{x x}^{j+\frac{1}{2}}\right) \\
= & -\left(u_{x x}^{j+\frac{1}{2}}-\frac{u_{x x}^{j}+u_{x x}^{j+1}}{2}, \bar{e}_{x x}^{j+\frac{1}{2}}\right)-\left(\frac{u_{x x}^{j+1}+u_{x x}^{j}}{2}-\frac{u_{N}^{j+1}+u_{N}^{j}}{2}, \bar{e}_{x x}^{j+\frac{1}{2}}\right) \\
\leq & \left\|u_{x x}^{j+\frac{1}{2}}-\frac{u_{x x}^{j}+u_{x x}^{j+1}}{2}\right\|\left\|\bar{e}_{x x}^{j+\frac{1}{2}}\right\|-\left(\bar{\eta}_{x x}^{j+\frac{1}{2}}+\bar{e}_{x x}^{j+\frac{1}{2}}, \bar{e}_{x x}^{j+\frac{1}{2}}\right) \\
\leq & \left(\frac{(\Delta t)^{3}}{96} \int_{t_{j}}^{t_{j+1}}\left\|u_{x x t t}\right\|^{2} d t\right)^{\frac{1}{2}}\left\|\bar{e}_{x x}^{j+\frac{1}{2}}\right\|-\left(\bar{\eta}_{x x}^{j+\frac{1}{2}}, \bar{e}_{x x}^{j+\frac{1}{2}}\right)-\left\|\bar{e}_{x x}^{j+\frac{1}{2}}\right\|^{2} . \\
\leq & \frac{(\Delta t)^{3}}{192} \int_{t_{j}}^{t_{j+1}}\left\|u_{x x t t}\right\|^{2} d t-\frac{1}{2}\left\|\bar{e}_{x x}^{j+\frac{1}{2}}\right\|^{2} .
\end{aligned}
$$

Then, Lemma 4 is proved.

Lemma 5. Assume that $u_{0} \in H_{E}^{2}(0,1), u$ is the solution for problem (1) and $u_{N}^{j}$ is the solution for problem (3.1). Then

$$
\begin{aligned}
& -\beta\left(u^{j+\frac{1}{2}}-\bar{u}_{N}^{j+\frac{1}{2}}, \bar{e}^{j+\frac{1}{2}}\right)-\alpha\left(u^{j+\frac{1}{2}}-\bar{u}_{N}^{j+\frac{1}{2}}, \bar{e}_{x x}^{j+\frac{1}{2}}\right) \\
\leq & \frac{k}{6}\left\|\bar{e}_{x x}^{j+\frac{1}{2}}\right\|^{2}+\frac{3 \alpha^{2}}{k}\left\|\bar{e}^{j+\frac{1}{2}}\right\|^{2}+c_{11} N^{-4},
\end{aligned}
$$

where $c_{11}$ is a positive constant depends only on $k, \alpha, \beta, \gamma, \delta, T$ and $\left\|u_{0}\right\|_{H_{E}^{2}}$, independent of $N$.

Proof. Noticing that

$$
\left\|\bar{\eta}^{j+\frac{1}{2}}\right\| \leq c\left(k, \alpha, \beta, \gamma, \delta, T,\left\|u_{0}\right\|_{H_{E}^{2}}\right) N^{-2} .
$$

Hence

$$
\begin{aligned}
& -\beta\left(u^{j+\frac{1}{2}}-\bar{u}_{N}^{j+\frac{1}{2}}, \bar{e}^{j+\frac{1}{2}}\right)-\alpha\left(u^{j+\frac{1}{2}}-\bar{u}_{N}^{j+\frac{1}{2}}, \bar{e}_{x x}^{j+\frac{1}{2}}\right) \\
= & -\beta\left(\bar{e}^{j+\frac{1}{2}}+\bar{\eta}^{j+\frac{1}{2}}, \bar{e}^{j+\frac{1}{2}}\right)-\alpha\left(\bar{e}^{j+\frac{1}{2}}+\bar{\eta}^{j+\frac{1}{2}}, \bar{e}_{x x}^{j+\frac{1}{2}}\right) \\
= & -\alpha\left\|\bar{e}^{j+\frac{1}{2}}\right\|\left\|\bar{e}_{x x}^{j+\frac{1}{2}}\right\|-\alpha\left\|\bar{\eta}^{j+\frac{1}{2}}\right\|\left\|\bar{e}_{x x}^{j+\frac{1}{2}}\right\|-\beta\left\|\bar{e}^{j+\frac{1}{2}}\right\|^{2}-\beta\left\|\bar{\eta}^{j+\frac{1}{2}}\right\|\left\|\bar{e}^{j+\frac{1}{2}}\right\| \\
\leq & \frac{k}{6}\left\|\bar{e}_{x x}^{j+\frac{1}{2}}\right\|^{2}+\frac{3 \alpha^{2}}{k}\left(\left\|\bar{e}^{j+\frac{1}{2}}\right\|^{2}+\left\|\bar{\eta}^{j+\frac{1}{2}}\right\|^{2}\right)+\frac{\beta}{4}\left\|\bar{\eta}^{j+\frac{1}{2}}\right\|^{2} \\
\leq & \frac{k}{6}\left\|\bar{e}_{x x}^{j+\frac{1}{2}}\right\|^{2}+\frac{3 \alpha^{2}}{k}\left\|\bar{e}^{j+\frac{1}{2}}\right\|^{2}+c_{11} N^{-4} .
\end{aligned}
$$

Then, Lemma 5 is proved. 
Lemma 6. Assume that $u_{0} \in H_{E}^{2}(0,1), u$ is the solution for problem (1) and $u_{N}^{j}$ is the solution for problem (3.1). Then

$$
\begin{aligned}
& -\gamma\left(\left(u_{x}^{j+\frac{1}{2}}\right)^{2}-\left(\bar{u}_{N x}^{j+\frac{1}{2}}\right)^{2}, \bar{e}^{j+\frac{1}{2}}\right) \\
\leq & \frac{k}{6}\left\|\bar{e}_{x x}^{j+\frac{1}{2}}\right\|^{2}+c_{12}\left(\frac{(\Delta t)^{3}}{96} \int_{t_{j}}^{t_{j+1}}\left\|u_{x t t}\right\|^{2} d t+\left\|\bar{e}^{j+\frac{1}{2}}\right\|^{2}+N^{-4}\right),
\end{aligned}
$$

where $c_{12}$ is a positive constant depends only on $k, \alpha, \beta, \gamma, \delta, T$ and $\left\|u_{0}\right\|_{H_{E}^{2}}$, independent of $N$.

Proof. Noticing that

$$
\begin{gathered}
\left\|\bar{e}_{N x}^{j+\frac{1}{2}}\right\|^{2} \leq \varepsilon\left\|\bar{e}_{N x x}^{j+\frac{1}{2}}\right\|^{2}+\frac{1}{4 \varepsilon}\left\|\bar{e}^{j+\frac{1}{2}}\right\|^{2}, \\
\left\|\bar{\eta}^{j+\frac{1}{2}}\right\| \leq c\left(k, \alpha, \beta, \gamma, \delta, T,\left\|u_{0}\right\|_{H_{E}^{2}}\right) N^{-2} .
\end{gathered}
$$

Hence, using the integration by parts, we have

$$
\begin{aligned}
& -\gamma\left(\left(u_{x}^{j+\frac{1}{2}}\right)^{2}-\left(\bar{u}_{N x}^{j+\frac{1}{2}}\right)^{2}, \bar{e}^{j+\frac{1}{2}}\right) \\
= & -\gamma\left(\left(u_{x}^{j+\frac{1}{2}}\right)^{2}-\left(\bar{u}_{x}^{j+\frac{1}{2}}\right)^{2}, \bar{e}^{j+\frac{1}{2}}\right)-\gamma\left(\left(\bar{u}_{x}^{j+\frac{1}{2}}\right)^{2}-\left(\bar{u}_{N x}^{j+\frac{1}{2}}\right)^{2}, \bar{e}^{j+\frac{1}{2}}\right) \\
= & -\gamma\left(\left(u_{x}^{j+\frac{1}{2}}+\bar{u}_{x}^{j+\frac{1}{2}}\right)\left(u_{x}^{j+\frac{1}{2}}-\bar{u}_{x}^{j+\frac{1}{2}}\right), \bar{e}^{j+\frac{1}{2}}\right) \\
& -\gamma\left(\left(\bar{u}_{x}^{j+\frac{1}{2}}+\bar{u}_{N x}^{j+\frac{1}{2}}\right)\left(\bar{e}_{x}^{j+\frac{1}{2}}+\bar{\eta}_{x}^{j+\frac{1}{2}}\right), \bar{e}^{j+\frac{1}{2}}\right) \\
= & -\gamma\left(\left(u_{x}^{j+\frac{1}{2}}+\bar{u}_{x}^{j+\frac{1}{2}}\right)\left(u_{x}^{j+\frac{1}{2}}-\bar{u}_{x}^{j+\frac{1}{2}}\right), \bar{e}^{j+\frac{1}{2}}\right) \\
& +\gamma\left(\bar{e}^{j+\frac{1}{2}}+\bar{\eta}^{j+\frac{1}{2}},\left(\bar{u}_{x x}^{j+\frac{1}{2}}+\bar{u}_{N x x}^{j+\frac{1}{2}}\right) \bar{e}^{j+\frac{1}{2}}+\left(\bar{u}_{x}^{j+\frac{1}{2}}+\bar{u}_{N x}^{j+\frac{1}{2}}\right) \bar{e}_{x}^{j+\frac{1}{2}}\right) .
\end{aligned}
$$

By Hölder's inequality and Sobolev's embedding theorem, we get

$$
\begin{aligned}
& \gamma\left(\left(u_{x}^{j+\frac{1}{2}}\right)^{2}-\left(\bar{u}_{N x}^{j+\frac{1}{2}}\right)^{2}, \bar{e}^{j+\frac{1}{2}}\right) \\
\leq & \gamma\left\|u_{x}^{j+\frac{1}{2}}+\bar{u}_{x}^{j+\frac{1}{2}}\right\|_{\infty}\left\|u_{x}^{j+\frac{1}{2}}-\bar{u}_{x}^{j+\frac{1}{2}}\right\|\left\|\bar{e}^{j+\frac{1}{2}}\right\| \\
& +\gamma\left(\left\|\bar{e}^{j+\frac{1}{2}}\right\|+\left\|\eta^{j+\frac{1}{2}}\right\|\right)\left\|\bar{u}_{x x}^{j+\frac{1}{2}}+\bar{u}_{N x x}^{j+\frac{1}{2}}\right\|\left\|\bar{e}^{j+\frac{1}{2}}\right\|_{\infty} \\
& +\gamma\left(\left\|\bar{e}^{j+\frac{1}{2}}\right\|+\left\|\eta^{j+\frac{1}{2}}\right\|\right)\left\|\bar{u}_{x}^{j+\frac{1}{2}}+\bar{u}_{N x}^{j+\frac{1}{2}}\right\|_{\infty}\left\|\bar{e}_{x}^{j+\frac{1}{2}}\right\| \\
\leq & c \gamma\left(\frac{(\Delta t)^{3}}{96} \int_{t_{j}}^{t_{j+1}}\left\|u_{x t t}\right\|^{2} d t\right)^{\frac{1}{2}}\left\|\bar{e}^{j+\frac{1}{2}}\right\|+c \gamma\left(\left\|\bar{e}^{j+\frac{1}{2}}\right\|+\left\|\eta^{j+\frac{1}{2}}\right\|\right)\left\|\bar{e}_{x}^{j+\frac{1}{2}}\right\| \\
\leq & \frac{k}{6}\left\|\bar{e}_{x x}^{j+\frac{1}{2}}\right\|^{2}+c_{12}\left(\frac{(\Delta t)^{3}}{96} \int_{t_{j}}^{t_{j+1}}\left\|u_{x t t}\right\|^{2} d t+\left\|\bar{e}^{j+\frac{1}{2}}\right\|^{2}+N^{-4}\right) .
\end{aligned}
$$


Hence, Lemma 6 is proved.

Lemma 7. Assume that $u_{0} \in H_{E}^{2}(0,1), u$ is the solution for problem (1) and $u_{N}^{j}$ is the solution for problem (3.1). Then

$$
\begin{aligned}
& -\delta\left(\left(u_{x}^{j+\frac{1}{2}}\right)^{3}-\left(\bar{u}_{N x}^{j+\frac{1}{2}}\right)^{3}, \bar{e}_{x}^{j+\frac{1}{2}}\right) \\
\leq & \frac{k}{6}\left\|\bar{e}_{x x}^{j+\frac{1}{2}}\right\|^{2}+c_{13}\left(\frac{(\Delta t)^{3}}{96} \int_{t_{j}}^{t_{j+1}}\left\|u_{x t t}\right\|^{2} d t+\left\|\bar{e}^{j+\frac{1}{2}}\right\|^{2}+N^{-4}\right) .
\end{aligned}
$$

where $c_{13}$ is a positive constant depends only on $k, \alpha, \beta, \gamma, \delta, T$ and $\left\|u_{0}\right\|_{H_{E}^{2}}$, independent of $N$.

Proof. Noticing that

$$
\left\|\bar{\eta}^{j+\frac{1}{2}}\right\| \leq c\left(k, \alpha, \beta, \gamma, \delta, T,\left\|u_{0}\right\|_{H_{E}^{2}}\right) N^{-2} .
$$

Hence, using the integration by parts, we have

$$
\begin{aligned}
& -\delta\left(\left(u_{x}^{j+\frac{1}{2}}\right)^{3}-\left(\bar{u}_{N x}^{j+\frac{1}{2}}\right)^{3}, \bar{e}_{x}^{j+\frac{1}{2}}\right) \\
= & -\delta\left(\left(u_{x}^{j+\frac{1}{2}}\right)^{3}-\left(\bar{u}_{x}^{j+\frac{1}{2}}\right)^{3}, \bar{e}_{x}^{j+\frac{1}{2}}\right)+\delta\left(\left(\bar{u}_{x}^{j+\frac{1}{2}}\right)^{3}-\left(\bar{u}_{N x}^{j+\frac{1}{2}}\right)^{3}, \bar{e}_{x}^{j+\frac{1}{2}}\right) \\
= & -\delta\left(\left(u_{x}^{j+\frac{1}{2}}+\bar{u}_{x}^{j+\frac{1}{2}}\right)\left(\left(u_{x}^{j+\frac{1}{2}}\right)^{2}+\left(\bar{u}_{x}^{j+\frac{1}{2}}\right)^{2}+\bar{u}_{x}^{j+\frac{1}{2}} \bar{u}_{N x}^{j+\frac{1}{2}}\right), \bar{e}_{x}^{j+\frac{1}{2}}\right) \\
& +\delta\left(\bar{u}^{j+\frac{1}{2}}-\bar{u}_{N}^{j+\frac{1}{2}},\right. \\
& \left.\left(2 \bar{u}_{x}^{j+\frac{1}{2}} \bar{u}_{x x}^{j+\frac{1}{2}}+\bar{u}_{x}^{j+\frac{1}{2}} \bar{u}_{N x x}^{j+\frac{1}{2}}+\bar{u}_{x x}^{j+\frac{1}{2}} \bar{u}_{N x}^{j+\frac{1}{2}}+2 \bar{u}_{N x} \bar{u}_{N x x}\right) \bar{e}_{x}^{j+\frac{1}{2}}\right) \\
+ & \left.\delta\left(\bar{u}^{j+\frac{1}{2}}-\bar{u}_{N}^{j+\frac{1}{2}},\left[\left(\bar{u}_{x}^{j+\frac{1}{2}}\right)^{2}+\bar{u}_{x}^{j+\frac{1}{2}} \bar{u}_{N x}^{j+\frac{1}{2}}+\left(\bar{u}_{N x}^{j+\frac{1}{2}}\right)^{2}\right]\right]_{x x}^{j+\frac{1}{2}}\right) .
\end{aligned}
$$

By Hölder's inequality and Sobolev's embedding theorem, we immediately obtain 


$$
\begin{aligned}
& -\delta\left(\left(u_{x}^{j+\frac{1}{2}}\right)^{3}-\left(\bar{u}_{N x}^{j+\frac{1}{2}}\right)^{3}, \bar{e}_{x}^{j+\frac{1}{2}}\right) \\
\leq & \delta\left\|\left(u_{x}^{j+\frac{1}{2}}\right)^{2}+\left(\bar{u}_{x}^{j+\frac{1}{2}}\right)^{2}+\bar{u}_{x}^{j+\frac{1}{2}} \bar{u}_{N x}^{j+\frac{1}{2}}\right\|_{\infty}\left\|u_{x}^{j+\frac{1}{2}}-\bar{u}_{x}^{j+\frac{1}{2}}\right\|\left\|\bar{e}_{x}^{j+\frac{1}{2}}\right\| \\
& +\delta\left(\left\|\bar{e}^{j+\frac{1}{2}}\right\|+\left\|\bar{\eta}^{j+\frac{1}{2}}\right\|\right) \| 2 \bar{u}_{x}^{j+\frac{1}{2}} \bar{u}_{x x}^{j+\frac{1}{2}}+\bar{u}_{x}^{j+\frac{1}{2}} \bar{u}_{N x x}^{j+\frac{1}{2}}+\bar{u}_{x x}^{j+\frac{1}{2}} \bar{u}_{N x}^{j+\frac{1}{2}} \\
& +2 \bar{u}_{N x} \bar{u}_{N x x}\|\| \bar{e}_{x}^{j+\frac{1}{2}} \|_{\infty} \\
& +\delta\left(\left\|\bar{e}^{j+\frac{1}{2}}\right\|+\left\|\bar{\eta}^{j+\frac{1}{2}}\right\|\right)\left\|\left(\bar{u}_{x}^{j+\frac{1}{2}}\right)^{2}+\bar{u}_{x}^{j+\frac{1}{2}} \bar{u}_{N x}^{j+\frac{1}{2}}+\left(\bar{u}_{N x}^{j+\frac{1}{2}}\right)\right\|_{\infty}\left\|\bar{e}_{x x}^{j+\frac{1}{2}}\right\| \\
\leq & c \delta\left(\frac{(\Delta t)^{3}}{96} \int_{t_{j}}^{t_{j+1}}\left\|u_{x t t}\right\|^{2} d t\right)^{\frac{1}{2}}\left\|\bar{e}^{j+\frac{1}{2}}\right\|+c \delta\left(\left\|\bar{e}^{j+\frac{1}{2}}\right\|+\left\|\eta^{j+\frac{1}{2}}\right\|\right)\left\|\bar{e}_{x x}^{j+\frac{1}{2}}\right\| \\
\leq & \frac{k}{6}\left\|\bar{e}_{x x}^{j+\frac{1}{2}}\right\|^{2}+c_{13}\left(\frac{(\Delta t)^{3}}{96} \int_{t_{j}}^{t_{j+1}}\left\|u_{x t t}\right\|^{2} d t+\left\|\bar{e}^{j+\frac{1}{2}}\right\|^{2}+N^{-4}\right) .
\end{aligned}
$$

Then, Lemma 7 is proved.

Thus, we obtain the following theorem.

Theorem 4. Assume that $u_{0} \in H_{E}^{2}(0,1), u(x, t)$ is the solution for problem (1) satisfying

$$
u \in L^{\infty}\left(0, T ; H_{E}^{2}(0,1)\right), u_{t t} \in L^{2}\left(0, T ; H_{E}^{2}(0,1)\right), u_{t t t} \in L^{2}\left(0, T ; L^{2}(0,1)\right) .
$$

Assume further that $u_{N}^{j}$ is the solution for problem (3.1). Then, if $\Delta t$ is sufficiently small, there exists positive constants $c_{14}$ depends on $k, \alpha, \beta, \gamma \delta, T,\left\|u_{0}\right\|_{H^{2}}$, independent of $N$, and $c_{15}$ depends on $k, \alpha, \beta, \gamma \delta, T,\left\|u_{0}\right\|_{H^{2}}, \int_{0}^{T}\left\|u_{t t}\right\|_{H^{2}}^{2} d t$, $\int_{0}^{T}\left\|u_{t t t}\right\|^{2} d t$, independent of $N$, such that, for $j=0,1,2, \cdots, N$,

$$
\left\|e^{j+1}\right\| \leq c_{14}\left(N^{-2}+\left\|e^{0}\right\|\right)+c_{15}(\Delta t)^{2} .
$$

Proof. By Lemmas 3-7, we obtain

$$
\begin{aligned}
\left\|e^{j+1}\right\|^{2} \leq & \left\|e^{j}\right\|^{2}+\Delta t c_{16}\left(\left\|e^{j+1}\right\|^{2}+\left\|e^{j}\right\|^{2}+N^{-4}\right) \\
& +(\Delta t)^{4} c_{17} \int_{t_{j}}^{t_{j+1}}\left(\left\|u_{t t}\right\|^{2}+\left\|u_{x t t}\right\|^{2}+\left\|u_{x x t t}\right\|^{2}+\left\|u_{t t t}\right\|^{2}\right) d t,
\end{aligned}
$$

where $c_{16}$ and $c_{17}$ are positive constants depend only on $k, a, b, T$ and $\left\|u_{0}\right\|_{H^{2}}$. For $\Delta t$ being sufficiently small, such that $c_{17} \Delta t \leq \frac{1}{2}$, setting $c_{18}=2\left(c_{16}+c_{17}\right)$, we get

$$
\left\|e^{j+1}\right\|^{2} \leq\left(1+c_{18} \Delta t\right)\left\|e^{j}\right\|^{2}+c_{18}\left(\Delta t N^{-4}+(\Delta t)^{4} B^{j}\right),
$$

where

$$
B^{j}=\int_{t_{j}}^{t_{j+1}}\left(\left\|u_{x t t}\right\|^{2}+\left\|u_{x x t t}\right\|^{2}+\left\|u_{t t t}\right\|^{2}\right) d t
$$


Using Gronwall's inequality for the discrete form, we have

$$
\left\|e^{j+1}\right\|^{2} \leq e^{c_{18}(j+1) \Delta t}\left(\left\|e^{0}\right\|^{2}+c_{18}\left(j \Delta t N^{-4}+(\Delta t)^{4} \sum_{i=0}^{j} B^{i}\right)\right) .
$$

Directly computation shows that

$$
\sum_{i=0}^{j} B^{i} \leq \int_{0}^{t_{j+1}}\left(\left\|u_{t t}\right\|^{2}+\left\|u_{x x t t}\right\|^{2}+\left\|u_{t t t}\right\|^{2}\right) d t .
$$

Thus, Theorem 4 is proved.

Furthermore, we have the following theorem.

Theorem 5. Assume that $u_{0} \in H_{E}^{2}(0,1), u(x, t)$ is the solution for problem (1) satisfying

$$
u \in L^{\infty}\left(0, T ; H_{E}^{2}(0,1)\right), u_{t t} \in L^{2}\left(0, T ; H_{E}^{2}(0,1)\right), u_{t t t} \in L^{2}\left(0, T ; L^{2}(0,1)\right) .
$$

Assume further that $u_{N}^{j} \in S_{N}(j=0,1,2, \cdots)$ is the solution for problem (3.1) and the initial value $u_{N}^{0}$ satisfies

$$
\left\|e^{0}\right\|=\left\|P_{N} u_{0}-u_{N}^{0}\right\| \leq c N^{-2}\left\|u_{x x}\right\| .
$$

Then, there exists positive constants $c^{\prime}$ depends on $k, \alpha, \beta, \gamma \delta, T,\left\|u_{0}\right\|_{H^{2}}$, independent of $N$, and $c^{\prime \prime}$ depends on $k, \alpha, \beta, \gamma \delta, T,\left\|u_{0}\right\|_{H^{2}}, \int_{0}^{T}\left\|u_{t t}\right\|_{H^{2}}^{2} d t, \int_{0}^{T}\left\|u_{t t t}\right\|^{2} d t$, independent of $N$, such that

$$
\left\|u\left(x, t_{j}\right)\right\|-u_{N}^{j} \| \leq c^{\prime} N^{-2}+c^{\prime \prime}(\Delta t)^{2}, \quad j=0,1,2, \cdots, N .
$$

\section{NUMERICAL RESULT}

In this section, using the Fourier spectral method described in (3.1), we carry out some numerical computations to illustrate out results in previous section. The fulldiscretization spectral method is read as: For $v_{l}=\sin l \pi x, l=1, \cdots, N$, find

$$
u_{N}^{n}=\sum_{i=0}^{N} \alpha_{i}^{n} \cos i \pi x \text {. }
$$

such that (3.1) hold.

As an example, we choose $k=1, \beta=2, \gamma=1, \alpha=1, \delta=1, u_{0}=(1-x)^{4} x^{4}+$ $0.001, \Delta t=0.001 / 2,0.001 / 4, N=32$, and get the solution which evolves from $t=0$ to $t=0.1$ (cf. Figure 1 ).

Now, we consider the variation of error. Since no exact solution to problem (1) is known for us, we make a comparison between the solution of (3.1) on a coarse mesh and on a fine mesh. 


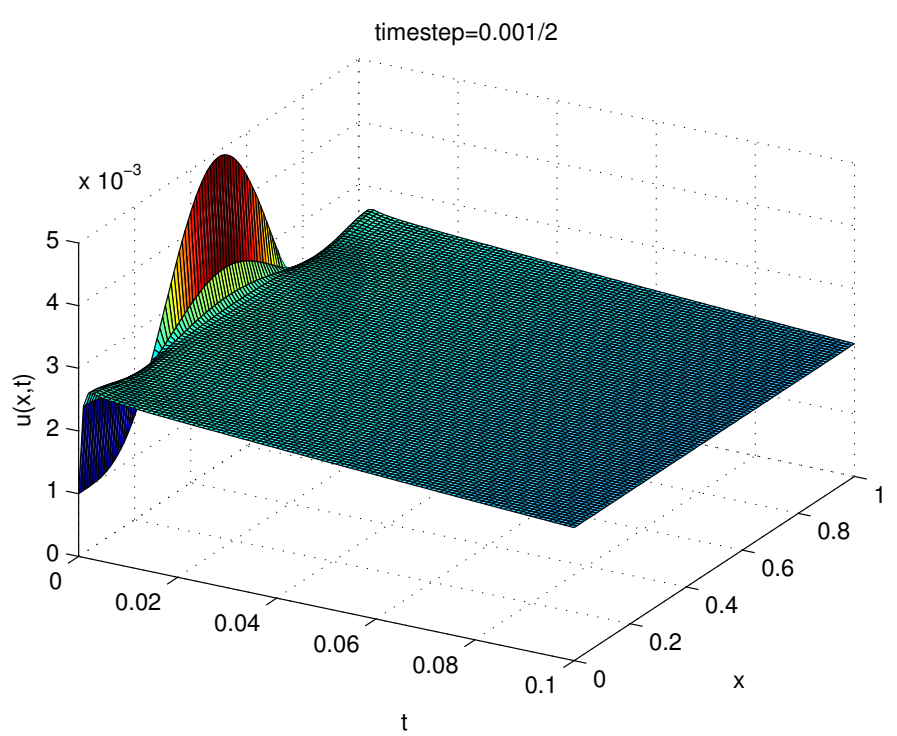

FIGURE 1 . The expanded property solution for $N=32, \Delta t=0.0005$.

We choose $\Delta t=0.001,0.001 \times \frac{1}{2}, 0.001 \times \frac{1}{4}, 0.001 \times \frac{1}{8}, 0.001 \times \frac{1}{16}$, respectively to solve (3.1). Set $u_{N}^{\min }(x, 0.1)$ as the solution for $\Delta t_{\min }=0.001 \times \frac{1}{32}$. Denote

$$
\operatorname{err}(0.1, \Delta t)=\left(\int_{0}^{1}\left(u_{N}^{k}(x, 0.1)-u_{N}^{\min }(x, 0.1)\right)^{2} d x\right)^{\frac{1}{2}}, k=1,2, \ldots, 6 .
$$

Then the error is showed in the Table 1 at $t=0.1$.

TABLE 1. The error for difference time step at $t=0.1$

\begin{tabular}{ccc}
\hline$\Delta t$ & $\operatorname{err}(0.1, \Delta t)$ & $\frac{\operatorname{err}(0.1, \Delta t)}{(\Delta t)^{2}}$ \\
\hline 0.001 & $2.3691 \times 10^{-6}$ & 2.3691 \\
\hline $0.001 \times \frac{1}{2}$ & $3.7397 \times 10^{-7}$ & 1.4959 \\
\hline $0.001 \times \frac{1}{4}$ & $5.6875 \times 10^{-8}$ & 0.9100 \\
\hline $0.001 \times \frac{1}{8}$ & $8.4589 \times 10^{-9}$ & 0.5413 \\
\hline $0.001 \times \frac{1}{16}$ & $1.0349 \times 10^{-9}$ & 0.1035 \\
\hline
\end{tabular}

In Table 1 , it is easy to see that the third column $\frac{\operatorname{err}(0.1, \Delta t)}{(\Delta t)^{2}}$ is monotone decreasing along with the time step's waning. Hence, we can find a positive constant $C=$ 2.3691 , such that

$$
\frac{\operatorname{err}(0.1, \Delta t)}{(\Delta t)^{2}} \leq C, k=1,2, \ldots, 5,
$$


which means the order of error estimates is $O\left((\Delta t)^{2}\right)$ proved in Theorem 5.

On the other hand, Choose $N=32,40,48,56, \Delta t_{0}=0.001 \times \frac{1}{32}$, respectively to solve (3.1).

TABLE 2. The errors of different basic function numbers at $\mathrm{t}=0.1$

\begin{tabular}{ccc}
\hline$N$ & $\operatorname{err}\left(0.1, \Delta t_{0}\right)$ & $\frac{\operatorname{err}\left(0.1, \Delta t_{0}\right)}{N^{-2}}$ \\
\hline 32 & $1.8637 \times 10^{-10}$ & $1.908 \times 10^{-7}$ \\
\hline 40 & $1.4862 \times 10^{-10}$ & $2.378 \times 10^{-7}$ \\
\hline 48 & $8.6941 \times 10^{-11}$ & $2.003 \times 10^{-7}$ \\
\hline 56 & $4.1655 \times 10^{-11}$ & $1.306 \times 10^{-7}$ \\
\hline
\end{tabular}

Then the error is showed in Table 2 at $t=0.1$. In Table 2 , it is easy to see that the third column $\frac{\operatorname{err}\left(0.1, \Delta t_{0}\right)}{(N)^{-2}}$ is almost monotone decreasing along with the time step's waning. Hence, we can find a positive constant $C=2.378 \times 10^{-7}$, such that

$$
\frac{\operatorname{err}(0.1, \Delta t)}{(\Delta t)^{2}} \leq C, N=32,40,48,56,
$$

which means the order of error estimates is $O\left(N^{-2}\right)$ proved in Theorem 5.

\section{CONCLUSiOnS}

Since the tools we have used work for the periodic boundary values, this result is also valid for the 1D Kolmogorov-Spiegel-Sivashinsky equation with the periodic boundary conditions. That is, for any $u_{0} \in H_{p e r}^{2}(0,1)$, choose the finite dimensional subspace of $H_{p e r}^{2}(0,1)$ :

$$
S_{N}=\operatorname{span}\left\{e^{i k x} ;-N / 2 \leq k \leq N / 2\right\},
$$

the existence, uniqueness and optimal error bonds for semi-discrete and fully discrete schemes can also be proved under the periodic boundary conditions

$$
\partial^{j} u(0, t)=\partial^{j} u(1, t), \quad t>0, j=0,1,2,3 .
$$

Since the original Kolmogorov-Spiegel-Sivashinsky(KSS) equation which arises in physical systems such as the Kolmogorov flow (a turbulent system of smallscale eddies supported by external energy sources) and the large-scale structure of compressible non-Boussinesqian convection (large scale turbulent solar convection) is formulated in $\mathbb{R}^{n}$. Here, we only consider the $1 \mathrm{D}$ case of the equation. If we want to understand the properties of this model better, we should study the numerical solutions for the multi-dimensional KSS equation, which is our intention in the future. 


\section{ACKNOWLEDGEMENTS}

The authors would like to express their deep thanks to the referee's valuable suggestions for the revision and improvement of the manuscript.

\section{REFERENCES}

[1] A. Biswas and D. Swanson, "Existence and generalized Gevrey regularity of solutions to the Kuramoto-Sivashinsky equation in $\mathbb{R}^{n}$," J. Differ. Equations, vol. 240, no. 1, pp. 145-163, 2007.

[2] C. Canuto, M. Hussaini, A. Quarteroni, and T. A. Zang, Spectral methods in fluid dynamics, ser. Springer Series in Computational Physics. New York: Springer-Verlag, 1988.

[3] S. Chai, Y. Zou, and C. Gong, "Spectral method for a class of Cahn-Hilliard equation with nonconstant mobility," Commun. Math. Res., vol. 25, no. 1, pp. 9-18, 2009.

[4] C. M. Elliott and S. Zheng, "On the Cahn-Hilliard equation," Arch. Ration. Mech. Anal., vol. 96, pp. 339-357, 1986.

[5] B. Guo and B. Wang, "Long-time behaviour of the solutions for the multidimensional Kolmogorov-Spiegel-Sivashinsky equation," Acta Math. Sin., Engl. Ser., vol. 18, no. 3, pp. 579596, 2002.

[6] Y. He and Y. Liu, "Stability and convergence of the spectral Galerkin method for the Cahn-Hilliard equation," Numer. Methods Partial Differ. Equations, vol. 24, no. 6, pp. 1485-1500, 2008.

[7] D. Michelson, "Steady solutions of the Kuramoto-Sivashinsky equation," Physica D, vol. 19, pp. 89-111, 1986.

[8] B. Nicolaenko, "Large scale spatial structures in two-dimensional turbulent flows," Nuclear Phys. $B$, vol. 2, pp. 453-484, 1987.

[9] B. Nicolaenko and B. Scheurer, "Remarks on the Kuramoto-Sivashinsky equation," Physica D, vol. 12, pp. 391-395, 1984.

[10] G. I. Sivashinsky, "Weak turbulence in periodic flows," Physica D, vol. 17, pp. 243-255, 1985.

[11] G. Ünal, "Principal resonances, local integrability and chaos in the Kolmogorov-SpiegelSivashinsky equation," Int. J. Eng. Sci., vol. 32, no. 3, pp. 455-472, 1994.

[12] G. Ünal and E. Şuhubi, "A local analysis of the Kolmogorov-Spiegel-Sivashinsky equation,” Int. J. Eng. Sci., vol. 30, no. 5, pp. 579-592, 1992.

[13] G. Ünal and E. Şuhubi, "Travelling waves and chaos in the Kolmogorov-Spiegel-Sivashinsky model," Int. J. Eng. Sci., vol. 30, no. 5, pp. 593-610, 1992.

[14] X. Xiang, The numerical analysis for spectral methods. Beijing: Science Press, 2000.

[15] T. Yang, "On traveling-wave solutions of the Kuramoto-Sivashinsky equation," Physica D, vol. 110, no. 1-2, pp. 25-42, 1997.

[16] X. Ye and X. Cheng, "The Fourier spectral method for the Cahn-Hilliard equation," Appl. Math. Comput., vol. 171, no. 1, pp. 345-357, 2005.

[17] X. Zhao, B. Liu, P. Zhang, W. Zhang, and F. Liu, Fourier spectral method for the modified SwiftHohenberg equation. Advances in Difference Equations, 2013.

[18] X. Zhao, M. Zhang, and C. Liu, "Some properties of solutions for a fourth-order parabolic equation,” Math. Methods Appl. Sci., vol. 36, no. 2, pp. 169-181, 2013.

Authors' addresses

Xiaopeng Zhao

Jiangnan University, School of Science, 214122 Wuxi, China

E-mail address: zxp032@126.com 
Bo Liu

Jilin University, College of Mathematics, 130012 Changchun, China

Peng Zhang

Chinese Academy of Sciences, Institute of Software, 100190 Beijing, China 